\title{
When do early-type galaxies form?
}

\author{
Roberto G. Abraham ${ }^{1}$, Patrick J. McCarthy ${ }^{2}$, Erin Mentuch ${ }^{1}$, \\ Karl Glazebrook ${ }^{3}$, Preethi Nair ${ }^{1}$, Jean-René Gauthier ${ }^{1}$, Sandra \\ Savaglio $^{4}$, David Crampton ${ }^{5}$, Stephanie Juneau ${ }^{5,6}$, Richard \\ Murowinski $^{5}$, Damien Le Borgne ${ }^{1}$, R. G. Carlberg ${ }^{1}$, Inger Jørgensen ${ }^{7}$, \\ Kathy Roth ${ }^{7}$, Hsiao-Wen Chen ${ }^{8}$, and Ronald O. Marzke ${ }^{9}$ \\ ${ }^{1}$ Department of Astronomy \& Astrophysics, University of Toronto \\ ${ }^{2}$ Observatories of the Carnegie Institution of Washington \\ ${ }^{3}$ Centre for Astrophysics and Supercomputing, Swinburne University of Technology \\ ${ }^{4}$ Max-Planck-Institut für extraterrestrische Physik \\ ${ }^{5}$ Herzberg Institute of Astrophysics \\ ${ }^{6}$ Steward Observatory, University of Arizona \\ ${ }^{7}$ Gemini Observatory \\ ${ }^{8}$ Dept. of Astronomy \& Astrophysics, University of Chicago \\ ${ }^{9}$ Dept. of Physics and Astronomy, San Francisco State University
}

\begin{abstract}
We have used the Hubble Space Telescope's Advanced Camera for Surveys to measure the mass density function of morphologically-selected early-type galaxies in the Gemini Deep Deep Survey fields, over the redshift range $0.9<z<1.6$. Our imaging data set covers four well-separated sight-lines, and is roughly intermediate (in terms of both depth and area) between the GOODS/GEMS imaging data, and the images obtained in the Hubble Deep Field campaigns. Our images contain 144 galaxies with ultra-deep spectroscopy, and they have been analyzed using a new purpose-written morphological analysis code which improves the reliability of morphological classifications by adopting a 'quasi-petrosian' image thresholding technique. We find that at $z=1$ approximately $70 \%$ of the stars in massive galaxies reside in early-type systems. This fraction is remarkably similar to that seen in the local Universe. However, we detect very rapid evolution in this fraction over the range $1.0<z<1.6$, suggesting that in this epoch the strong color-morphology relationship seen in the nearby Universe is beginning to fall into place.
\end{abstract}

Keywords. galaxy evolution, galaxy formation, morphology

\section{Introduction}

The era at which a strong correlation between galaxy morphology and stellar content develops is not well constrained. One of the primary goals of the Gemini Deep Deep Survey (GDDS; Abraham et al. 2004) was to use stellar-mass-selected samples to probe galaxy evolution in the critical $1<z<2$ range, over which something like half the stellar mass in galaxies is expected to form (based upon integration of the cosmic starformation history presented in Steidel et al. 1999). The GDDS and other samples (e.g. K20; Cimatti et al. 2004) showed that the total stellar mass density evolves slowly for $z<1.5$ and that the high-mass end in particular is slowly evolving (Glazebrook et al. 2004; Fontana et al. 2004). In this short paper we examine the evolution of the stellar mass density as a function of morphological type using deep HST/ACS imaging of the GDDS fields. We outline how a slight refinement (based on adopting a so-called 'quasipetrosian' threshold) to traditional methods allows morphological classification based on central concentration (or Gini coefficent) vs. asymmetry. Using this method, we are able 
to derive robust morphological classifications at $1<z<2$, and present the stellar mass function for early-type galaxies over this redshift range.

\section{Quasi-Petrosian measurements}

The benefits of determining galaxy properties within a metric size defined by a circular Petrosian aperture are well-established (Petrosian 1976; Blanton et al. 2001), so we will not describe them here except to note that galaxy properties determined in this way are quite robust to the effects of cosmological dimming and bandshifting. What is less wellknown is that a 'Quasi-Petrosian' aperture can also be constructed using an algorithm which works for galaxies of arbitrary shape†. Interestingly, this algorithm is closely related to the pixel-sorting algorithm used to define the Gini coefficient (Abraham et al. 2003), which itself provides an elegant and robust proxy for central concentration and which is proving useful for galaxy classification (Abraham et al. 2003, Lotz et al. 2004) .

A 'quasi-Petrosian aperture' can be defined in the following manner. All pixels in the galaxy image are sorted in decreasing order of flux to construct an array, $f_{i}$, containing the flux in the $i$ th sorted pixel. This array is then summed over to construct a monotonically increasing curve of cumulative flux values:

$$
\mathcal{F}_{i}=\sum_{j=1}^{i} f_{j} .
$$

In analogy with the definition of the Petrosian radius for a circular aperture, a 'Petrosian isophote' can be calculated by determining the pixel index $i$ which satisfies the following equation:

$$
f_{i}=q \times\left(\frac{\mathcal{F}_{i}}{i}\right)
$$

The flux value of the pixel which solves this equation is used to construct the quasipetrosian aperture mask. In fact, once $f_{i}$ is known, constructing the aperture mask is trivial: pixels brighter than $f_{i}$ are set to 1.0, and pixels fainter than $f_{i}$ are set to 0.0 . Morphological quantities are computed in the usual way after multiplying the original image by the aperture mask. Measurements obtained through non-circular quasi-Petrosian apertures share many of the salutary properties of photometric measurements obtained through circular Petrosian apertures. The free parameter $q$ in the equation is fixed for all galaxies in the sample, and ideally all groups using this approach would adopt the same value of $q$ to allow easy inter-comparison of measurements. A little experimentation has shown us that $q=0.2$ yields excellent results, and we suggest that this be adopted as a sensible value. Finally, we note that because the data are discrete, Equation 2.2 will rarely be solved exactly, but a perfectly adequate approximation can be obtained by simply noting the index of the first zero crossing of $f_{i}-q \times\left(\frac{\mathcal{F}_{i}}{i}\right)$.

\section{The evolving mass-density function of Early-type galaxies}

In Figure 1 we show quasi-Petrosian aperture-based measurements of Asymmetry vs. Gini coefficent for all galaxies in with $I<23.5 \mathrm{mag}$ in our GDDS ACS images (Abraham

$\dagger$ We learned in Prague that Ned Taylor at Leiden Observatory has also been experimenting with quasi-petrosian statistics, which he defines using equations similar to those given in this section.

$\ddagger$ Public-domain software for undertaking morphological measurements is available at this URL: http://odysseus.astro.utoronto.ca/ abraham/Morpheus/ 


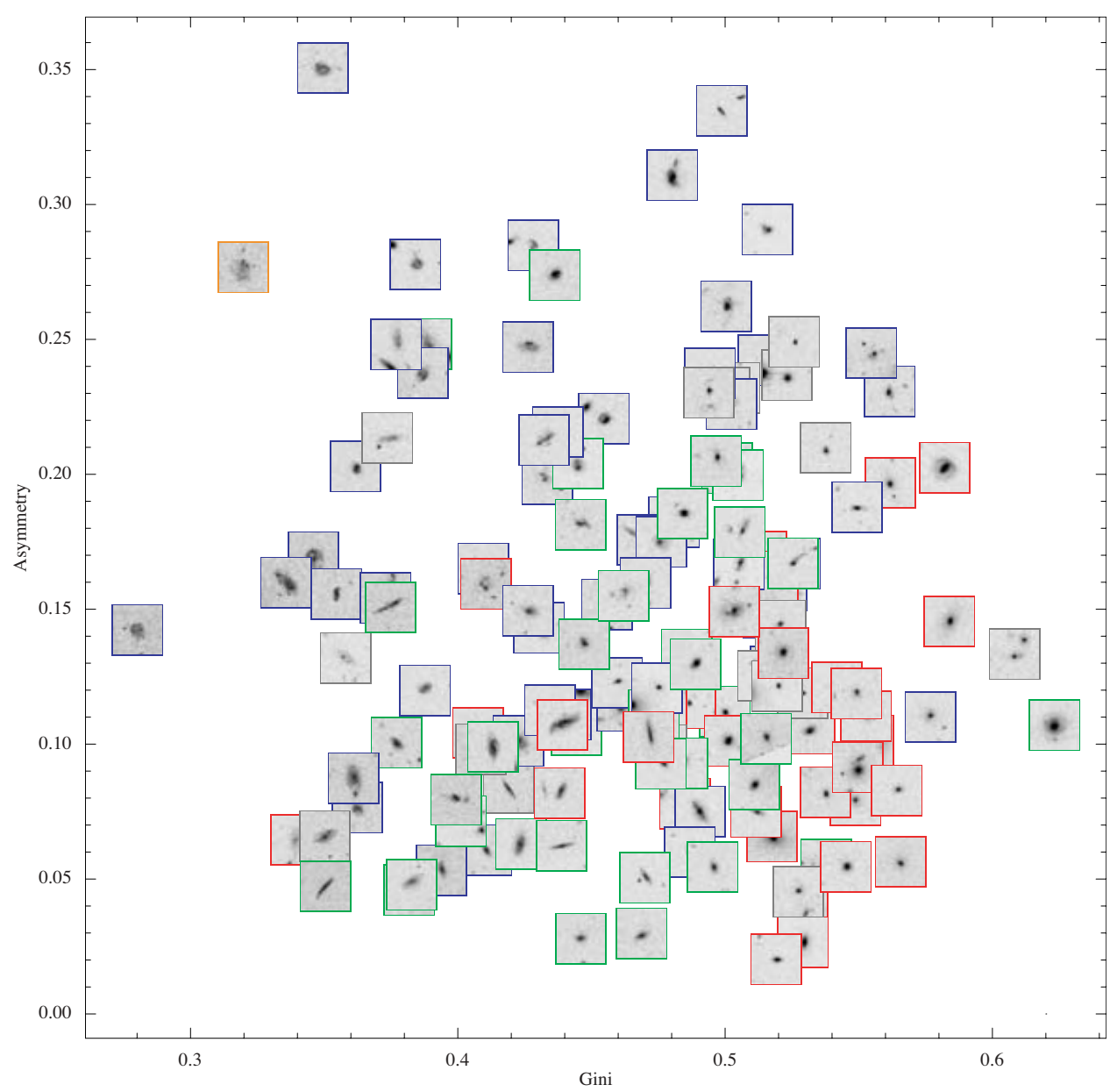

Figure 1. Asymmetry vs. Gini for galaxies in the Gemini Deep Deep Survey. Individual galaxies are shown as four arcsec 'postage stamps', whose borders are colored according to spectral classification. Red borders correspond to galaxies dominated by older stellar populations.

et al. 2006). Inspection of this plot (ideally in electronic form so zooming-in on the small postage stamp images is possible!) shows that this two-dimensional classification plane does a good job of distinguishing early-type galaxies from all other galaxy types. Interestingly, a comparison of the morphological mix with the spectral classifications of the galaxies shows that surprisingly little ambiguity exists in connecting spectral classes to morphological classes for quiescent systems. This can be seen in the electronic version of this paper, which shows this figure using colored borders on the galaxy images to denote spectroscopic classification.

Figure 2 shows the evolving stellar mass density function for massive $\left(\log _{10}\left(M / M_{\odot}\right)>\right.$ 10.5) galaxies in our ACS images, computed using the formalism described in the previous section. If we restrict consideration to massive galaxies only, at $z=1$, about $70 \%$ of the stellar mass in these systems lives in early-type galaxies. This is essentially identical to the local value (Abraham et al. 2006). Interestingly, we see strong evolution in this fraction over the range $1<z<1.6$, as the mass densities in spheroids evolve steeply over this redshift range. These results strongly suggest that the core formation epoch for massive spheroids is drawing to a close in this interval. Signatures of major mergers 


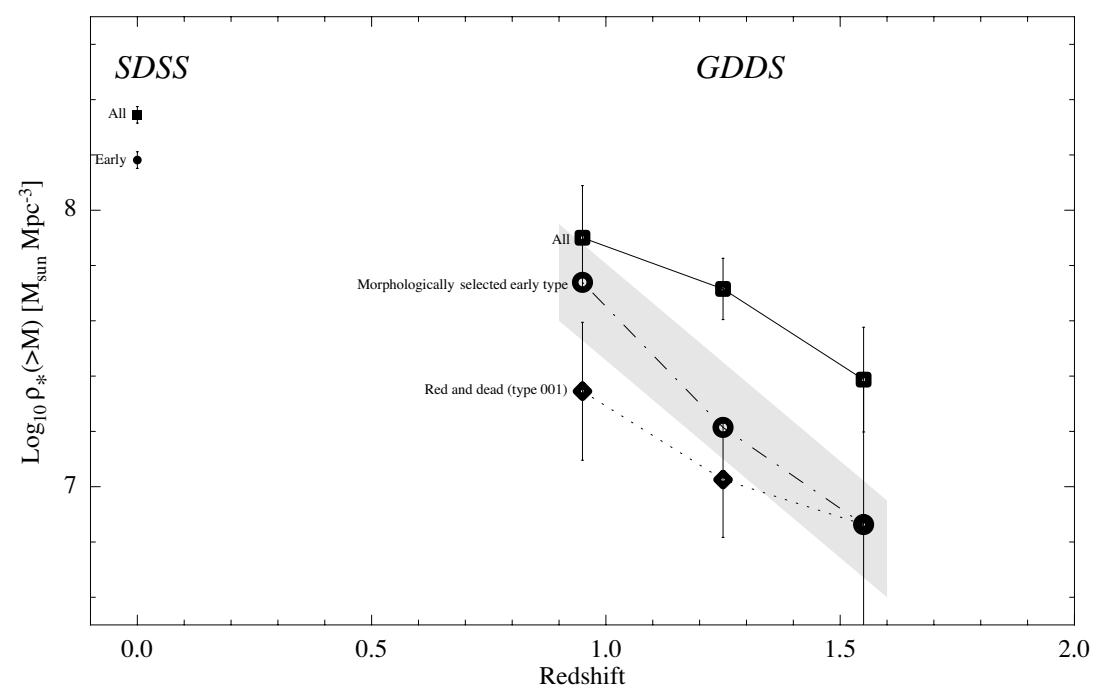

Figure 2. Mass density functions for subsets of massive galaxies, defined as having stellar masses $>10^{10.5} M_{\odot}$. Plot symbols denote the following subsets: spectroscopically quiescent 'Red and dead' galaxies (diamonds); morphologically selected early-type galaxies (circles); and all galaxies (squares). The gray band corresponds to the approximate uncertainty in the early-type measurment. The local stellar mass densities for massive galaxies, taken from the analysis of SDSS observations given by Bell et al. (2004) and converted to our IMF and mass cut, are also shown.

appear to have faded by $z \sim 1.5$ or higher, and subsequent mass evolution at $z<1$ apparently does not significantly disturb the spectral-morphology correlation.

\section{Acknowledgements}

We thank Francoise Combes for her indulgence in accepting this manuscript even though it was late in coming, and extend our congratulations to the local organizers of the IAU symposium in Prague for hosting an interesting meeting.

\section{References}

Abraham, R. G., van den Bergh, S. \& Nair, P., (2003). ApJ, 588, 218

Abraham, R. G., Glazebrook, K., McCarthy, P. J., Crampton, D., Murowinski, R. et al., 2004, $A J, 127,2455$

Bell, E. F., et al. 2004, ApJ, 608, 752

Blanton et al. 2001, AJ, 121, 2358

Cimatti, A., Daddi, E., Renzini, A., Cassata, P., Vanzella, E. et al. 2004, Nature, 430, 184

Fontana, A., Pozzetti, L., Donnarumma, I., Renzini, A., Cimmatti, A. et al. 2004, A\&A, 424, 23

Glazebrook, K., Blake, C., Economou, F., Lilly, S., Colless, M., 1999, MNRAS, 306, 843

Glazebrook, K., Abraham, R. G., Savaglio, S., McCarthy, P.J.P., Crampton, D. et al., 2004, Nature, 430, 181

Lotz, J., Primack, J. \& Madau, P. 2004, AJ, 128, 163

McCarthy, P. J., Le Borgne, D., Crampton, D., Chen, H.-W., Abraham, R. G. et al. 2004, ApJ, 614, L9

Petrosian, v. 1976. ApJ, 209L, 1P

Steidel, C. C., Adelgerger, K. L., Giavalisco, M., Dickinson, M., Pettini, M., 1999, ApJ, 519, 1. 


\section{Discussion}

Chris Conselice: A comment: When you talk about the comparison between the Gini index and the concentration index, it is important to be clear about which index you are using. Graham et al. (2001) clearly show that the Abraham concentration index is very unstable when measured at high redshift, while others such as those based on the ratio of radii are much better and can be used for automatic classifications.

RoBerto ABRAHAM: OK 